\title{
Quaternary Hermitian linear complementary dual codes
}

\author{
Makoto Araya*, Masaaki Harada ${ }^{\dagger} a n d$ Ken Saito ${ }^{\ddagger}$ \\ January 1, 2020
}

\begin{abstract}
The largest minimum weights among quaternary Hermitian linear complementary dual codes are known for dimension 2. In this paper, we give some conditions on the nonexistence of quaternary Hermitian linear complementary dual codes with large minimum weights. As a consequence, we completely determine the largest minimum weights for dimension 3 , by using a classification of some quaternary codes. In addition, for a positive integer $s$, an entanglement-assisted quantum error-correcting [[21s $+5,3,16 s+3 ; 21 s+2]]$ code with maximal entanglement is constructed for the first time from a quaternary Hermitian linear complementary dual $[26,3,19]$ code.
\end{abstract}

\section{Introduction}

Linear complementary dual (LCD for short) codes are linear codes that intersect with their dual trivially. LCD codes were introduced by Massey [14] and gave an optimum linear coding solution for the two user binary adder channel.

*Department of Computer Science, Shizuoka University, Hamamatsu 432-8011, Japan. email: araya@inf.shizuoka.ac.jp

${ }^{\dagger}$ Research Center for Pure and Applied Mathematics, Graduate School of Information Sciences, Tohoku University, Sendai 980-8579, Japan. email: mharada@tohoku.ac.jp.

${ }^{\ddagger}$ Research Center for Pure and Applied Mathematics, Graduate School of Information Sciences, Tohoku University, Sendai 980-8579, Japan. email: kensaito@ims.is.tohoku.ac.jp. 
Recently, much work has been done concerning LCD codes for both theoretical and practical reasons (see e.g. 1], [5], 6], 8, [11, [12] and the references given therein). For example, if there is a quaternary Hermitian LCD $[n, k, d]$ code, then there is a maximal entanglement $[[n, k, d ; n-k]]$ entanglementassisted quantum error-correcting code (EAQECC for short) (see e.g. [1] and [12]). From this point of view, quaternary Hermitian LCD codes play an important role in the study of maximal entanglement EAQECC's.

It is a fundamental problem to determine the largest minimum weight $d_{4}(n, k)$ among all quaternary Hermitian LCD $[n, k]$ codes for a given pair $(n, k)$. It was shown that $d_{4}(n, 2)=\left\lfloor\frac{4 n}{5}\right\rfloor$ if $n \equiv 1,2,3(\bmod 5)$ and $d_{4}(n, 2)=$ $\left\lfloor\frac{4 n}{5}\right\rfloor-1$ if $n \equiv 0,4(\bmod 5)$ for $n \geq 3$ in [10] and [12]. In this paper, we give some conditions on the nonexistence of quaternary Hermitian LCD codes with large minimum weights. We give a classification of (unrestricted) quaternary $[4 r, 3,3 r]$ codes for $r=9,10,12,13,14,16$ and quaternary $[43,3,32]$ codes. Using the above classification and the classification in [3], we completely determine the largest minimum weight among all quaternary Hermitian LCD codes of dimension 3. In addition, for a positive integer $s$, a maximal entanglement $[[21 s+5,3,16 s+3 ; 21 s+2]]$ EAQECC is constructed for the first time from a quaternary Hermitian LCD [26, 3, 19] code.

This paper is organized as follows. In Section 2, we prepare some definitions, notations and basic results used in this paper. In Section 3, we give characterizations of quaternary Hermitian LCD codes. It is shown that there is no quaternary Hermitian LCD $\left[\frac{4^{k}-1}{3} s, k, 4^{k-1} s\right]$ code for $k \geq 3$ and $s \geq 1$ (Theorem 3.3). In addition, if $4\left(4^{k-1} n-\frac{4^{k}-1}{3} \alpha\right)<k$, where $k \geq 3$ and $4 \alpha-3 n \geq 1$, then there is no quaternary Hermitian LCD $[n, k, \alpha]$ code $C$ with $d\left(C^{\perp_{H}}\right) \geq 2$, where $d(C)$ denotes the minimum (Hamming) weight of a quaternary code $C$ and $C^{\perp_{H}}$ denotes the Hermitian dual code of $C$. If $4\left(4^{k-1} n-\frac{4^{k}-1}{3} \alpha\right) \geq k \geq 3$, where $4 \alpha-3 n \geq 1$ and there is no quaternary Hermitian LCD $\left[4\left(4^{k-1} n-\frac{4^{k}-1}{3} \alpha\right), k, 3\left(4^{k-1} n-\frac{4^{k}-1}{3} \alpha\right)\right]$ code $C_{0}$ with $d\left(C_{0}^{\perp_{H}}\right) \geq 2$, then there is no quaternary Hermitian LCD $[n, k, \alpha]$ code $C$ with $d\left(C^{\perp_{H}}\right) \geq 2$ (Theorem 3.4). In Section 4, from the classification of quaternary codes of dimension 3 by Bouyukliev, Grassl and Varbanov [3], we determine $d_{4}(n, 3)$ for $n \leq 35$. We emphasize that there is a quaternary Hermitian LCD $[26,3,19]$ code. This implies the existence of a quaternary Hermitian LCD $[21 s+5,3,16 s+3]$ code for $s \geq 1$ (Proposition 5.4). We also give a classification of quaternary $[4 r, 3,3 r]$ codes for $r=9,10,12,13,14,16$ and quaternary $[43,3,32]$ codes. In Section 5, we completely determine $d_{4}(n, 3)$ 
(Theorem 5.1). This result is mainly obtained by applying Theorems 3.3 and 3.4 to the classification of some quaternary codes of dimension 3 given in Section 4. As a consequence of Proposition 5.4, we show that there is a maximal entanglement $[[21 s+5,3,16 s+3 ; 21 s+2]]$ EAQECC for $s \geq 1$. This determines the largest minimum weight among maximal entanglement $[[21 s+5,3, d ; 21 s+2]]$ EAQECC's as $16 s+3$ (Remark [5.5). Finally, in Appendix, we give a proof of Proposition 2.4.

\section{Preliminaries}

In this section, we prepare some definitions, notations and basic results used in this paper.

\subsection{Definitions and notations}

We denote the finite field of order 4 by $\mathbb{F}_{4}=\left\{0,1, \omega, \omega^{2}\right\}$, where $\omega^{2}=\omega+$ 1. For any element $\alpha \in \mathbb{F}_{4}$, the conjugation of $\alpha$ is defined as $\bar{\alpha}=\alpha^{2}$. Throughout this paper, we use the following notations. Let $\mathbf{0}_{s}$ and $\mathbf{1}_{s}$ denote the zero vector and the all-one vector of length $s$, respectively. Let $O$ denote the zero matrix of appropriate size. Let $I_{k}$ denote the identity matrix of order $k$ and let $A^{T}$ denote the transpose of a matrix $A$. For a matrix $A=\left(a_{i j}\right)$, the conjugate matrix of $A$ is defined as $\bar{A}=\left(\overline{a_{i j}}\right)$. For a $k \times n$ matrix $A$, we denote by $A^{(s)}$ the $k \times n s$ matrix $\left(\begin{array}{lll}A & \cdots & A\end{array}\right)$.

A quaternary $[n, k]$ code $C$ is a $k$-dimensional vector subspace of $\mathbb{F}_{4}^{n}$. A generator matrix of a quaternary $[n, k]$ code $C$ is a $k \times n$ matrix such that the rows of the matrix generate $C$. The weight wt $(x)$ of a vector $x \in \mathbb{F}_{4}^{n}$ is the number of non-zero components of $x$. A vector of $C$ is called a codeword of $C$. The minimum non-zero weight of all codewords in $C$ is called the minimum weight $d(C)$ of $C$. A quaternary $[n, k, d]$ code is a quaternary $[n, k]$ code with minimum weight $d$. The weight enumerator of a quaternary $[n, k]$ code $C$ is the polynomial $\sum_{i=0}^{n} A_{i} y^{i}$, where $A_{i}$ denotes the number of codewords of weight $i$ in $C$. Two quaternary $[n, k]$ codes $C$ and $C^{\prime}$ are equivalent if there is an $n \times n$ monomial matrix $P$ over $\mathbb{F}_{4}$ with $C^{\prime}=\{x P \mid x \in C\}$.

For any (unrestricted) quaternary $[n, k, d]$ code, the Griesmer bound is given by

$$
n \geq \sum_{i=0}^{k-1}\left\lceil\frac{d}{4^{i}}\right\rceil .
$$


Throughout this paper, we use the following notation:

$$
\alpha_{4}(n, k)=\max \left\{d \in \mathbb{Z}_{\geq 0} \mid n \geq \sum_{i=0}^{k-1}\left\lceil\frac{d}{4^{i}}\right\rceil\right\},
$$

where $\mathbb{Z}_{\geq 0}$ denotes the set of nonnegative integers.

The Euclidean dual code $C^{\perp}$ of a quaternary $[n, k]$ code $C$ is defined as $C^{\perp}=\left\{x \in \mathbb{F}_{4}^{n} \mid\langle x, y\rangle=0\right.$ for all $\left.y \in C\right\}$, where $\langle x, y\rangle=\sum_{i=1}^{n} x_{i} y_{i}$ for $x=\left(x_{1}, \ldots, x_{n}\right), y=\left(y_{1}, \ldots, y_{n}\right) \in \mathbb{F}_{4}^{n}$. The Hermitian dual code $C^{\perp_{H}}$ of a quaternary $[n, k]$ code $C$ is defined as $C^{\perp_{H}}=\left\{x \in \mathbb{F}_{4}^{n} \mid\langle x, y\rangle_{H}=0\right.$ for all $y \in$ $C\}$, where $\langle x, y\rangle_{H}=\sum_{i=1}^{n} x_{i} \overline{y_{i}}$ for $x=\left(x_{1}, \ldots, x_{n}\right), y=\left(y_{1}, \ldots, y_{n}\right) \in \mathbb{F}_{4}^{n}$. A quaternary code $C$ is called Euclidean linear complementary dual if $C \cap C^{\perp}=$ $\left\{\mathbf{0}_{n}\right\}$. A quaternary code $C$ is called Hermitian linear complementary dual if $C \cap C^{\perp_{H}}=\left\{\mathbf{0}_{n}\right\}$. These two families of quaternary codes are collectively called linear complementary dual (LCD for short) codes. Note that quaternary Hermitian LCD codes are also called zero radical codes (see e.g. [11] and [12]).

A quaternary code $C$ is called Hermitian self-orthogonal if $C \subset C^{\perp_{H}}$. A quaternary code $C$ is called even if the weights of all codewords of $C$ are even. It is known that a quaternary code $C$ is Hermitian self-orthogonal if and only if $C$ is even [13, Theorem 1]. In addition, a quaternary code $C$ is Hermitian self-orthogonal if and only if $G \bar{G}^{T}=O$ for a generator matrix $G$ of $C$.

A $2-(v, k, \lambda)$ design $\mathcal{D}$ is a pair of a set $\mathcal{P}$ of $v$ points and a collection of $k$-element subsets of $\mathcal{P}$ (called blocks) such that every 2-element subset of $\mathcal{P}$ is contained in exactly $\lambda$ blocks. The number of blocks that contain a given point is traditionally denoted by $r$, and the total number of blocks is $b$. Often a $2-(v, k, \lambda)$ design is simply called a 2-design. A 2-design is called symmetric if $v=b$. A 2-design can be represented by its incidence matrix $A=\left(a_{i j}\right)$, where $a_{i j}=1$ if the $j$-th point is contained in the $i$-th block and $a_{i j}=0$ otherwise.

\subsection{Quaternary Hermitian LCD codes}

The following characterization gives a criterion for quaternary Hermitian LCD codes and is analogous to [14, Proposition 1].

Proposition 2.1 ([8, Proposition 3.5]). Let $C$ be a quaternary code. Let $G$ 
be a generator matrix of $C$. Then $C$ is Hermitian $L C D$ if and only if $G \bar{G}^{T}$ is nonsingular.

Throughout this paper, we use the above characterization without mentioning this.

Lemma 2.2. Suppose that there is a quaternary Hermitian $L C D[n, k, d]$ code $C$. If $d_{4}(n-1, k) \leq d-1$, then $d\left(C^{\perp_{H}}\right) \geq 2$.

Proof. Suppose that $d\left(C^{\perp_{H}}\right)=1$. Then some column of a generator matrix of $C$ is $\mathbf{0}_{k}^{T}$. By deleting this column, a quaternary Hermitian LCD $[n-1, k, d]$ code is constructed. This contradicts the assumption that $d_{4}(n-1, k) \leq$ $d-1$.

Lemma 2.3. Let $G_{1}$ and $G_{2}$ be generator matrices of a quaternary Hermitian $L C D\left[n_{1}, k, d_{1}\right]$ code and a quaternary Hermitian self-orthogonal $\left[n_{2}, k, d_{2}\right]$ code, respectively. Then the code with generator matrix $\left(\begin{array}{lll}G_{1} & G_{2}\end{array}\right)$ is a quaternary Hermitian $L C D\left[n_{1}+n_{2}, k, d^{\prime}\right]$ code with $d^{\prime} \geq d_{1}+d_{2}$.

Proof. The straightforward proof is omitted.

\subsection{Determination of $d_{4}(n, 2)$}

Suppose that there is an (unrestricted) quaternary $[n, 2, d]$ code. By the Griesmer bound (11), we have

$$
d \leq\left\lfloor\frac{4 n}{5}\right\rfloor \text {. }
$$

Lu, Li, Guo and Fu [12, Lemma 3.1] constructed a quaternary Hermitian LCD $\left[n, 2,\left\lfloor\frac{4 n}{5}\right\rfloor\right]$ code for $n \equiv 1,2,3(\bmod 5)$ and $n \geq 3$, and a quaternary Hermitian LCD $\left[n, 2,\left\lfloor\frac{4 n}{5}\right\rfloor-1\right]$ code for $n \equiv 0,4(\bmod 5)$ and $n \geq 4$. The following proposition is mentioned in [12, by quoting [10].

Proposition 2.4 ( $\mathrm{Li}[10])$. If $n \equiv 0,4(\bmod 5)$, then there is no quaternary Hermitian $L C D\left[n, 2,\left\lfloor\frac{4 n}{5}\right\rfloor\right]$ code.

Remark 2.5. In Appendix, we give a proof of the above proposition for the sake of completeness.

Hence, one can determine $d_{4}(n, 2)$ as follows:

$$
d_{4}(n, 2)= \begin{cases}\left\lfloor\frac{4 n}{5}\right\rfloor & \text { if } n \equiv 1,2,3 \quad(\bmod 5), \\ \left\lfloor\frac{4 n}{5}\right\rfloor-1 & \text { if } n \equiv 0,4 \quad(\bmod 5),\end{cases}
$$

for $n \geq 3$. 


\section{Nonexistence of some quaternary Hermi- tian LCD codes}

In this section, we give results on the nonexistence of some quaternary Hermitian LCD codes.

An easy counting argument yields the following lemma. We give a proof for the sake of completeness. Recall that $b$ and $r$ denote the number of blocks of a $2-(v, k, \lambda)$ design $\mathcal{D}$ and the number of blocks containing a given point of $\mathcal{D}$, respectively.

Lemma 3.1. Let $n$ and $\alpha$ be positive integers. Let $m=\left(m_{1}, \ldots, m_{v}\right)$ be a vector of $\mathbb{Z}_{\geq 0}^{v}$. Suppose that there is a $2-(v, k, \lambda)$ design $\mathcal{D}$. Let $A$ be the incidence matrix of $\mathcal{D}$. If each entry of the $b \times 1 \mathbb{Z}$-matrix $A m^{T}$ is at least $\alpha$, then

$$
\frac{r \alpha-\lambda \sum_{j=1}^{v} m_{j}}{r-\lambda} \leq m_{i} \leq \sum_{j=1}^{v} m_{j}-\frac{b-r}{r-\lambda} \alpha
$$

for any $i \in\{1, \ldots, v\}$.

Proof. Fix a point $p$ of $\mathcal{D}$. Define the following sets:

$$
X_{0}=\left\{i \in\{1, \ldots, b\} \mid a_{i p}=0\right\} \text { and } X_{1}=\left\{i \in\{1, \ldots, b\} \mid a_{i p}=1\right\},
$$

where $A=\left(a_{i j}\right)$. Let $w_{i}$ denote the $i$-th entry of the $b \times 1 \mathbb{Z}$-matrix $A m^{T}$. Then we have

$$
\sum_{i \in X_{1}} w_{i}=r m_{p}+\sum_{j \in\{1, \ldots, v\} \backslash\{p\}} \lambda m_{j}=(r-\lambda) m_{p}+\lambda \sum_{j=1}^{v} m_{j} .
$$

Since $\sum_{i \in X_{0}} w_{i}+\sum_{i \in X_{1}} w_{i}=\sum_{i=1}^{b} w_{i}=r \sum_{j=1}^{v} m_{j}$, we have

$$
\sum_{i \in X_{0}} w_{i}=(r-\lambda) \sum_{j=1}^{v} m_{j}-(r-\lambda) m_{p}
$$

Since $\left|X_{1}\right|=r$ and $\left|X_{0}\right|=b-r$, we have

$$
r \alpha \leq \sum_{i \in X_{1}} w_{i} \text { and }(b-r) \alpha \leq \sum_{i \in X_{0}} w_{i}
$$

This completes the proof. 
According to [12, we define the $k \times\left(\frac{4^{k}-1}{3}\right)$ matrices $S_{k}$ by inductive constructions as follows:

$$
\begin{aligned}
& S_{1}=(1), \\
& S_{k}=\left(\begin{array}{ccccc}
S_{k-1} & \mathbf{0}_{\frac{4^{k-1}-1}{3}}^{T} & S_{k-1} & S_{k-1} & S_{k-1} \\
\mathbf{0}_{\frac{4_{k-1}-1}{3}}^{1} & \mathbf{1}_{\frac{4^{k-1}-1}{3}} & \omega \mathbf{1}_{\frac{4^{k-1}-1}{3}} & \omega^{2} \mathbf{1}_{\frac{4^{k-1}-1}{3}}
\end{array}\right) \text { if } k \geq 2 .
\end{aligned}
$$

The matrix $S_{k}$ is a generator matrix of the quaternary $\left[\frac{4^{k}-1}{3}, k, 4^{k-1}\right]$ simplex code. It is known that the quaternary $\left[\frac{4^{k}-1}{3}, k, 4^{k-1}\right]$ simplex code is a constant weight code. More precisely, the code contains codewords of weights 0 and $4^{k-1}$ only. Thus, for $k \geq 2$, the quaternary $\left[\frac{4^{k}-1}{3}, k, 4^{k-1}\right]$ simplex code is even. By [13, Theorem 1], the quaternary $\left[\frac{4^{k}-1}{3}, k, 4^{k-1}\right]$ simplex code is Hermitian self-orthogonal for $k \geq 2$.

Let $h_{k}^{(i)}$ be the $i$-th column of $S_{k}$. For a vector $m=\left(m_{1}, \ldots, m_{\frac{4^{k}-1}{3}}\right) \in$ $\mathbb{Z}_{\geq 0}^{\frac{4^{k}-1}{3}}$ with $\sum_{i} m_{i}=n$, we define a matrix:

$$
G_{k}(m)=(\underbrace{h_{k}^{(1)} \cdots h_{k}^{(1)}}_{m_{1} \text { columns }} \cdots \underbrace{h_{k}^{\left(\frac{4^{k}-1}{3}\right)} \cdots h_{k}^{\left(\frac{4^{k}-1}{3}\right)}}_{m_{\frac{4^{k}-1}{3}} \text { columns }}) .
$$

For a quaternary $[n, k]$ code $C$ with $d\left(C^{\perp_{H}}\right) \geq 2$, there is a vector $m=$ $\left(m_{1}, \ldots, m_{\frac{4^{k}-1}{3}}\right) \in \mathbb{Z}_{\geq 0}^{\frac{4^{k}-1}{3}}$ such that $C$ is equivalent to a code with generator matrix $G_{k}(m)$. We denote the code by $C_{k}(m)$.

Lemma 3.2. Suppose that $k \geq 3, m=\left(m_{1}, \ldots, m_{\frac{4^{k}-1}{3}}\right) \in \mathbb{Z}_{\geq 0}^{\frac{4^{k}-1}{3}}$ and $\sum_{i} m_{i}=n$. If a quaternary $[n, k]$ code $C_{k}(m)$ has minimum weight at least $\alpha$, then

$$
4 \alpha-3 n \leq m_{i} \leq n-\frac{4^{k-1}-1}{3 \cdot 4^{k-2}} \alpha
$$

for any $i \in\left\{1, \ldots, \frac{4^{k}-1}{3}\right\}$.

Proof. It is well known that the supports of the codewords of weight $4^{k-1}$ in the quaternary $\left[\frac{4^{k}-1}{3}, k, 4^{k-1}\right]$ simplex code form a symmetric $2-\left(\frac{4^{k}-1}{3}, 4^{k-1}, 3\right.$. $4^{k-2}$ ) design for $k \geq 3$ (see e.g. [4, p. 8]). As the 2-design $\mathcal{D}$ in Lemma $\underline{3.1}$, 
consider the symmetric $2-\left(\frac{4^{k}-1}{3}, 4^{k-1}, 3 \cdot 4^{k-2}\right)$ design. Since $d\left(C_{k}(m)\right) \geq \alpha$, it follows from the structure of $C_{k}(m)$ that each entry of the $\frac{4^{k}-1}{3} \times 1$ matrix $A m^{T}$ is at least $\alpha$. The result follows from Lemma 3.1.

If we write $n=\frac{4^{k}-1}{3} s$, then $\alpha_{4}(n, k)=4^{k-1} s$ (see (2) for $\alpha_{4}(n, k)$ ).

Theorem 3.3. Suppose that $k \geq 3$. If $n \equiv 0\left(\bmod \frac{4^{k}-1}{3}\right)$, then there is no quaternary Hermitian $L C D\left[n, k, \alpha_{4}(n, k)\right]$ code.

Proof. Write $n=\frac{4^{k}-1}{3} s$. Suppose that there is a quaternary Hermitian LCD $\left[\frac{4^{k}-1}{3} s, k, 4^{k-1} s\right]$ code $C$. Since $\alpha_{4}\left(\frac{4^{k}-1}{3} s-1, k\right) \leq 4^{k-1} s-1, d\left(C^{\perp_{H}}\right) \geq 2$ by Lemma 2.2. Thus, we may assume that $C$ is equivalent to a code $C_{k}(m)$ for some vector $m=\left(m_{1}, \ldots, m_{\frac{4^{k}-1}{3}}\right) \in \mathbb{Z}_{\geq 0}^{\frac{4^{k}-1}{3}}$. Consider the conditions given in (44). Since we have

$$
4^{k} s-3\left(\frac{4^{k}-1}{3} s\right)=s \text { and } \frac{4^{k}-1}{3} s-\frac{4^{k-1}-1}{3 \cdot 4^{k-2}} 4^{k-1} s=s,
$$

by Lemma [3.2, we have $m_{i}=s$ for each $i$. This means that $m=s \mathbf{1}_{\frac{4^{k}-1}{3}}$. Since $S_{k}{\overline{S_{k}}}^{T}=O$, we have

$$
G_{k}\left(s \mathbf{1}_{\frac{4^{k}-1}{3}}\right){\overline{G_{k}\left(s \mathbf{1}_{\frac{4^{k}-1}{3}}\right.}}^{T}=S_{k}^{(s)}{\overline{S_{k}^{(s)}}}^{T}=O .
$$

By [13, Theorem 1], $C_{k}(m)$ is quaternary Hermitian self-orthogonal, which is a contradiction.

Set

$$
r_{4}(n, k, \alpha)=4^{k-1} n-\frac{4^{k}-1}{3} \alpha,
$$

for positive integers $n, k$ and $\alpha$. The following theorem is one of the main results in this paper.

Theorem 3.4. Suppose that $k \geq 3$ and $4 \alpha-3 n \geq 1$.

(i) Suppose that $4 r_{4}(n, k, \alpha)<k$. Then there is no quaternary Hermitian $L C D[n, k, \alpha]$ code $C$ with $d\left(C^{\perp_{H}}\right) \geq 2$.

(ii) Suppose that $4 r_{4}(n, k, \alpha) \geq k$. If there is no quaternary Hermitian $L C D$ $\left[4 r_{4}(n, k, \alpha), k, 3 r_{4}(n, k, \alpha)\right]$ code $C_{0}$ with $d\left(C_{0}^{\perp_{H}}\right) \geq 2$, then there is no quaternary Hermitian $L C D[n, k, \alpha]$ code $C$ with $d\left(C^{\perp_{H}}\right) \geq 2$. 
Proof. Suppose that there is a quaternary Hermitian LCD $[n, k, \alpha]$ code $C$ with $d\left(C^{\perp_{H}}\right) \geq 2$. Then $C$ is equivalent to a code $C_{k}(m)$ with generator matrix $G_{k}(m)$ for some vector $m=\left(m_{1}, \ldots, m_{\frac{4^{k}-1}{3}}\right) \in \mathbb{Z}_{\geq 0}^{\frac{4^{k}-1}{3}}$. Since $d(C)=$ $\alpha$, by Lemma 3.2 , we have

$$
4 \alpha-3 n \leq m_{i},
$$

for each $i \in\left\{1, \ldots, \frac{4^{k}-1}{3}\right\}$. Thus, at least $4 \alpha-3 n$ columns of the matrix $G_{k}(m)$ are $h_{k}^{(i)}$, then we obtain a matrix $G$ of the following form:

$$
G=\left(\begin{array}{cc}
G_{0} & S_{k}^{(4 \alpha-3 n)}
\end{array}\right)
$$

by permuting columns of $G_{k}(m)$. Here, $G_{0}$ is a $k \times n_{0}$ matrix, where

$$
n_{0}=4\left(4^{k-1} n-\frac{4^{k}-1}{3} \alpha\right)=4 r_{4}(n, k, \alpha) \text {. }
$$

The code $C^{\prime}$ with generator matrix $S_{k}^{(4 \alpha-3 n)}$ is a quaternary Hermitian selforthogonal $\left[n^{\prime}, k, d^{\prime}\right]$ code, where

$$
n^{\prime}=(4 \alpha-3 n) \frac{4^{k}-1}{3} \text { and } d^{\prime}=(4 \alpha-3 n) 4^{k-1} .
$$

Since $S_{k}{\overline{S_{k}}}^{T}=O$, we have $G \bar{G}^{T}=G_{0}{\overline{G_{0}}}^{T}$. Since $\operatorname{rank}\left(G \bar{G}^{T}\right)=k$, we have

$$
\operatorname{rank}\left(G_{0}\right) \geq \operatorname{rank}\left(G_{0}{\overline{G_{0}}}^{T}\right)=k .
$$

(i) Suppose that $4 r_{4}(n, k, \alpha)<k$. Since $G_{0}$ is a $k \times 4 r_{4}(n, k, \alpha)$ matrix,

$$
k>4 r_{4}(n, k, \alpha) \geq \operatorname{rank}\left(G_{0}\right) \geq k,
$$

which is a contradiction.

(ii) Suppose that $4 r_{4}(n, k, \alpha) \geq k$. Let $C_{0}$ be the quaternary code with generator matrix $G_{0}$. Since $G \bar{G}^{T}=G_{0}{\overline{G_{0}}}^{T}, C_{0}$ is a quaternary Hermitian LCD $\left[n_{0}, k\right]$ code. It follows from the form of $G_{0}$ that $d\left(C_{0}^{\perp_{H}}\right) \geq 2$. Let $d_{0}$ denote the minimum weight of $C_{0}$. By Lemma 2.3, $\alpha \geq d_{0}+d^{\prime}$. Since $C^{\prime}$ is a constant weight code, there is a codeword of weight $d_{0}+d^{\prime}$ in $C_{k}(m)$. Thus, $\alpha=d_{0}+d^{\prime}$ then we have

$$
d_{0}=-\left(4^{k}-1\right) \alpha+4^{k-1} 3 n=3 r_{4}(n, k, \alpha) .
$$

Therefore, there is a quaternary Hermitian $\operatorname{LCD}\left[4 r_{4}(n, k, \alpha), k, 3 r_{4}(n, k, \alpha)\right]$ code $C_{0}$ with $d\left(C_{0}^{\perp_{H}}\right) \geq 2$. 
This completes the proof.

Remark 3.5. If $4 \alpha-3 n \geq 1$, then we have

$$
n \geq 4 r_{4}(n, k, \alpha)+\frac{4^{k}-1}{3},
$$

since $n-4 r_{4}(n, k, \alpha) \geq \frac{1}{3}\left(-3 n\left(4^{k}-1\right)+(3 n+1)\left(4^{k}-1\right)\right)=\frac{1}{3}\left(4^{k}-1\right)$.

\section{Quaternary codes of dimension 3}

In this section, a classification of (unrestricted) quaternary codes of dimension 3 is done for some lengths by using computer calculations (see Lemma 5.6 for the motivation of our classification). All computer calculations were done by programs in MAgma [2] and programs in the language C.

\subsection{Classification method}

A shortened code $C^{\prime}$ of a quaternary code $C$ is the set of all codewords in $C$ which are 0 in a fixed coordinate with that coordinate deleted. A shortened code $C^{\prime}$ of a quaternary $[n, k, d]$ code $C$ with $d \geq 2$ is a quaternary $[n-1, k, d]$ code if the deleted coordinate is a zero coordinate and a quaternary $\left[n-1, k-1, d^{\prime}\right]$ code with $d^{\prime} \geq d$ otherwise.

By considering the inverse operation of shortening, every quaternary $[n, 3, d]$ code with $d \geq 2$ is constructed from some quaternary $\left[n-1,2, d^{\prime}\right]$ code with $d^{\prime} \geq d$. By considering equivalent quaternary codes, we may assume that a quaternary $\left[n-1,2, d^{\prime}\right]$ code has the following generator matrix:

$$
\left(\begin{array}{cc|cc|cccc}
1 & 0 & \mathbf{0}_{a_{1}} & \mathbf{0}_{a_{2}} & \mathbf{1}_{a_{3}} & \mathbf{1}_{a_{4}} & \mathbf{1}_{a_{5}} & \mathbf{1}_{a_{6}} \\
0 & 1 & \mathbf{0}_{a_{1}} & \mathbf{1}_{a_{2}} & \mathbf{0}_{a_{3}} & \mathbf{1}_{a_{4}} & \omega \mathbf{1}_{a_{5}} & \omega^{2} \mathbf{1}_{a_{6}}
\end{array}\right),
$$

where $a_{1}+a_{2}=n-d^{\prime}-2$ and $a_{3}+a_{4}+a_{5}+a_{6}=d^{\prime}-1$. For the generator matrix (5) of each of all inequivalent quaternary $\left[n-1,2, d^{\prime}\right]$ codes with $d^{\prime} \geq d$, consider the generator matrices $\left(\begin{array}{ll}I_{3} & M\end{array}\right)$, where

$$
M=\left(\begin{array}{cc|cccc}
\mathbf{0}_{a_{1}} & \mathbf{0}_{a_{2}} & \mathbf{1}_{a_{3}} & \mathbf{1}_{a_{4}} & \mathbf{1}_{a_{5}} & \mathbf{1}_{a_{6}} \\
\mathbf{0}_{a_{1}} & \mathbf{1}_{a_{2}} & \mathbf{0}_{a_{3}} & \mathbf{1}_{a_{4}} & \omega \mathbf{1}_{a_{5}} & \omega^{2} \mathbf{1}_{a_{6}} \\
x_{1} & x_{2} & x_{3} & x_{4} & x_{5} & x_{6}
\end{array}\right),
$$


where $x_{i}=\left(x_{i, 1}, \ldots, x_{i, a_{i}}\right)$, under the condition that $x_{i, j} \leq x_{i, k}$ for $j<k$ and $x_{1, \ell} \in\{0,1\}$. Here, we consider a natural order on the elements of $\mathbb{F}_{4}$ as follows $0<1<\omega<\omega^{2}$. In this way, all quaternary $[n, 3, d]$ codes, which must be checked further for equivalences, are constructed. By checking equivalences among these codes, we complete a classification of quaternary $[n, 3, d]$ codes.

\subsection{Lengths up to 35}

Here we investigate the values $d_{4}(n, 3)$ for $n=4,5, \ldots, 35$. Let $d_{4}^{\text {all }}(n, 3)$ denote the largest minimum weight among all (unrestricted) quaternary $[n, 3]$ codes (see [7] for the current information on $d_{4}^{\text {all }}(n, 3)$ ). Lu, Li, Guo and $\mathrm{Fu}$ [12 found quaternary Hermitian LCD codes having large minimum weights for dimension 3. From [12, Tables 3 and 4], we know $d_{4}(n, 3)=$ $d_{4}^{\text {all }}(n, 3)$ for

$$
n=7,8,9,10,11,12,13,17,18,23,24,25,28,29,30,33,34 .
$$

For $n \leq 35$, Bouyukliev, Grassl and Varbanov [3] completed the classification of (unrestricted) quaternary $\left[n, 3, d_{4}^{\text {all }}(n, 3)\right]$ codes. The number of the inequivalent quaternary $\left[n, 3, d_{4}^{\text {all }}(n, 3)\right]$ codes are given in [3, Table 3]. Based on the number given in [3, Table 3], we reconstructed all inequivalent quaternary $\left[n, 3, d_{4}^{\text {all }}(n, 3)\right]$ codes for

$$
n=4,5,6,14,15,16,19,20,21,22,26,27,31,32,35,
$$

by using the method in Section 4.1. Then we found that $d_{4}(n, 3)<d_{4}^{\text {all }}(n, 3)$ for the above lengths except 26. For length 26, we found that $d_{4}(n, 3)=$ $d_{4}^{\text {all }}(n, 3)$. For the remaining lengths, from [12, Tables 3 and 4], we know $d_{4}(n, 3)=d_{4}^{\text {all }}(n, 3)-1$. This determines the largest minimum weight $d_{4}(n, 3)$ for lengths $n=4,5, \ldots, 35$, where the results are listed in Table 1. In the table, the reference about the existence of quaternary Hermitian LCD $\left[n, 3, d_{4}(n, 3)\right]$ codes is also listed.

We give details for the case $d_{4}(26,3)=19$. There are five inequivalent quaternary $[26,3,19]$ codes $[3$, Table 3$]$. We verified that one of them is 
Table 1: $d_{4}(n, 3)(n=4,5, \ldots, 35)$

\begin{tabular}{|c|c|c|c|c|c|}
\hline$n$ & $d_{4}(n, 3)$ & Reference & $n$ & $d_{4}(n, 3)$ & Reference \\
\hline 4 & 1 & {$[12$, Table 3] } & 20 & 14 & {$[12$, Table 3] } \\
\hline 5 & 2 & [12, Table 3] & 21 & 15 & {$[12$, Table 3] } \\
\hline 6 & 3 & [12, Table 3] & 22 & 15 & {$[12$, Table 4] } \\
\hline 7 & 4 & [12, Table 3] & 23 & 16 & {$[12$, Table 4] } \\
\hline 8 & 5 & [12, Table 3] & 24 & 17 & [12, Table 4] \\
\hline 9 & 6 & [12, Table 3] & 25 & 18 & {$[12$, Table 4] } \\
\hline 10 & 6 & {$[12$, Table 3] } & 26 & 19 & $C_{26}$ \\
\hline 11 & 7 & [12, Table 3] & 27 & 19 & {$[12$, Table 4] } \\
\hline 12 & 8 & [12, Table 3] & 28 & 20 & {$[12$, Table 4] } \\
\hline 13 & 9 & {$[12$, Table 3] } & 29 & 21 & {$[12$, Table 4] } \\
\hline 14 & 9 & [12, Table 3] & 30 & 22 & {$[12$, Table 4] } \\
\hline 15 & 10 & {$[12$, Table 3] } & 31 & 22 & {$[12$, Table 4] } \\
\hline 16 & 11 & [12, Table 3] & 32 & 23 & [12, Table 4] \\
\hline 17 & 12 & {$[12$, Table 3] } & 33 & 24 & {$[12$, Table 4] } \\
\hline 18 & 13 & {$[12$, Table 3] } & 34 & 25 & {$[12$, Table 4] } \\
\hline 19 & 13 & {$[12$, Table 3] } & 35 & 25 & [12, Table 4] \\
\hline
\end{tabular}

Hermitian LCD. This code $C_{26}$ has the following generator matrix:

$$
\left(\begin{array}{cccccccccccccccccc}
1 & 0 & 0 & 0 & 0 & 0 & 0 & 0 & 1 & 1 & 1 & 1 & 1 & & & & & \\
0 & 1 & 0 & 0 & 1 & 1 & 1 & 1 & 0 & 0 & 0 & 0 & 1 & & & & & \\
0 & 0 & 1 & 1 & 1 & 1 & \omega & \omega & 1 & 1 & \omega & \omega^{2} & 0 \\
& & & & & 1 & 1 & 1 & 1 & 1 & 1 & 1 & 1 & 1 & 1 & 1 & 1 & 1 \\
& & & & & 1 & 1 & 1 & 1 & \omega & \omega & \omega & \omega & \omega^{2} & \omega^{2} & \omega^{2} & \omega^{2} & \omega^{2} \\
& & & & & 1 & \omega & \omega & \omega^{2} & 0 & 1 & \omega & \omega^{2} & 0 & 1 & \omega & \omega^{2} & \omega^{2}
\end{array}\right),
$$

and the following weight enumerator:

$$
1+33 y^{19}+18 y^{20}+3 y^{21}+9 y^{22} .
$$

\subsection{Lengths $36,40,43,48,52,56$ and 64}

By using the method in Section 4.1, a classification of (unrestricted) quaternary $[4 r, 3,3 r]$ codes for $r=9,10,12,13,14,16$ and quaternary [43, 3, 32] 
codes was done. These codes have minimum weights $d_{4}^{\text {all }}(n, 3)$. To save space, the results are given only.

Proposition 4.1. (i) There are two inequivalent quaternary $[4 r, 3,3 r]$ codes $C_{4 r, i}(i=1,2)$, none of which is Hermitian $L C D$ for $r=9,10$.

(ii) There are ten inequivalent quaternary $[43,3,32]$ codes $C_{43, i}(i=1,2, \ldots, 10)$, none of which is Hermitian LCD.

(iii) There are five inequivalent quaternary $[4 r, 3,3 r]$ codes $C_{4 r, i}(i=1,2, \ldots, 5)$, none of which is Hermitian LCD for $r=12,13$.

(iv) There are six inequivalent quaternary $[56,3,42]$ codes $C_{56, i}(i=1,2, \ldots, 6)$, none of which is Hermitian LCD.

(v) There are 15 inequivalent quaternary $[64,3,48]$ codes $C_{64, i}(i=1,2, \ldots, 15)$, none of which is Hermitian LCD.

Table 2: Vectors $v_{j}^{T}(j=1,2, \ldots, 22)$

\begin{tabular}{c|c|c|c|c|c|c|c|c|c}
\hline$i$ & $v_{i}^{t}$ & $i$ & $v_{i}^{T}$ & $i$ & $v_{i}^{T}$ & $i$ & $v_{i}^{T}$ & $i$ & $v_{i}^{T}$ \\
\hline 1 & $(0,0,1)$ & 6 & $\left(0,1, \omega^{2}\right)$ & 11 & $(1,1,0)$ & 16 & $(1, \omega, 1)$ & 21 & $\left(1, \omega^{2}, \omega\right)$ \\
2 & $(0,0,1)$ & 7 & $(1,0,0)$ & 12 & $(1,1,1)$ & 17 & $(1, \omega, \omega)$ & 22 & $\left(1, \omega^{2}, \omega^{2}\right)$ \\
3 & $(0,1,0)$ & 8 & $(1,0,1)$ & 13 & $(1,1, \omega)$ & 18 & $\left(1, \omega, \omega^{2}\right)$ & & \\
4 & $(0,1,1)$ & 9 & $(1,0, \omega)$ & 14 & $\left(1,1, \omega^{2}\right)$ & 19 & $\left(1, \omega^{2}, 0\right)$ & & \\
5 & $(0,1, \omega)$ & 10 & $\left(1,0, \omega^{2}\right)$ & 15 & $(1, \omega, 0)$ & 20 & $\left(1, \omega^{2}, 1\right)$ & & \\
\hline
\end{tabular}

In order to display the matrices $M$ in (6) for generator matrices $\left(\begin{array}{ll}I_{3} & M\end{array}\right)$ of $C_{4 r, i}(r=9,10,12,13,14,16)$ and $C_{43, i}$, we give some vectors $v_{i}^{T}$ of length 3 in Table 2. Let $n_{j}(j=1,2, \ldots, 22)$ be the number of the columns of $M$ in (6), which are equal to $v_{j}$. The numbers $n_{j}(j=1,2, \ldots, 22)$ are listed in Tables 3 and 4 . The weight enumerators $W_{n, i}$ of $C_{n, i}$ are listed in Table 5 .

\section{$5 \quad$ Determination of $d_{4}(n, 3)$}

The aim of this section is to establish the following theorem, which is one of the main results in this paper. 
Table 3: $\left(n_{1}, n_{2}, \ldots, n_{22}\right)$

\begin{tabular}{c|c}
\hline Code & $\left(n_{1}, n_{2}, \ldots, n_{22}\right)$ \\
\hline$C_{36,1}$ & $(0,1,0,2,2,2,0,2,2,2,1,2,2,2,0,2,2,2,1,2,2,2)$ \\
$C_{36,2}$ & $(0,1,0,2,2,2,0,2,2,2,1,2,2,2,1,1,2,2,1,2,2,2)$ \\
\hline$C_{40,1}$ & $(0,0,1,2,2,2,1,2,2,2,2,2,2,2,2,1,2,2,2,2,2,2)$ \\
$C_{40,2}$ & $(0,1,1,2,2,2,1,2,0,2,2,2,2,2,2,2,2,2,2,2,2,2)$ \\
\hline
\end{tabular}

Theorem 5.1. Suppose that $n \geq 6$. Then

$$
d_{4}(n, 3)=\left\{\begin{array}{cc}
\left\lfloor\frac{16 n}{21}\right\rfloor & \text { if } n \equiv 5,9,13,17,18 \quad(\bmod 21) \\
\left\lfloor\frac{16 n}{21}\right\rfloor-1 & \text { if } n \equiv 0,1,2,3,4,6,7,8, \\
10,11,12,14,15,16,19,20 \quad(\bmod 21) .
\end{array}\right.
$$

Remark 5.2. For $n \equiv 2,3,4,8,9,12,13,17,18(\bmod 21)$, the above result is known [12, Table 4].

Suppose that there is an (unrestricted) quaternary $[n, 3, d]$ code. Write $n=21 s+t$, where $0 \leq t \leq 20$. By the Griesmer bound (1), we have $d \leq \alpha_{4}(n, 3)$, where $\alpha_{4}(n, 3)$ are listed in Table 6 for each $n=21 s+t(s \geq 1$ if $t=0,1,2$ and $s \geq 0$ if $t=3,4, \ldots, 20)$.

Lemma $5.3(\mathrm{Lu}, \mathrm{Li}, \mathrm{Guo}$ and $\mathrm{Fu}[12])$. If there is a quaternary Hermitian $L C D[n, 3, d]$ code, then there is a quaternary Hermitian $L C D[21 s+n, 3,16 s+$ d] code for every positive integer $s$.

By the Griesmer bound (11) and [12, Table 4], we have

$$
d_{4}(21 s+5,3)=16 s+2 \text { or } 16 s+3 .
$$

The code $C_{26}$ given in Section 4.2 is a quaternary Hermitian LCD $[26,3,19]$ code. By Lemma 5.3, there is a quaternary Hermitian LCD $\left[21 s^{\prime}+26,3,16 s^{\prime}+\right.$ 19] code for a positive integer $s^{\prime}$. Therefore, we have the following:

Proposition 5.4. For a positive integer $s, d_{4}(21 s+5,3)=16 s+3$.

Remark 5.5. An $[[n, k, d ; c]]$ EAQECC $\mathcal{C}$ encodes $k$ information qubits into $n$ channel qubits with the help of $c$ pairs of maximally entangled Bell states. The parameter $d$ is called the minimum weight of $\mathcal{C}$. The EAQECC $\mathcal{C}$ can correct up to $\left\lfloor\frac{d-1}{2}\right\rfloor$ errors acting on the $n$ channel qubits (see e.g. [11] and [12]). 
Table 4: $\left(n_{1}, n_{2}, \ldots, n_{22}\right)$

\begin{tabular}{|c|c|}
\hline Code & $\left(n_{1}, n_{2}\right.$, \\
\hline$C_{43,1}$ & $0,1,2,3,3,0,1,1,1,2,3,3,2,2,3,3,2,2,2,2$ \\
\hline$C_{43,2}$ & $0,0,3,3,3,1,2,2,2,2,2,2,2,2,2,2,2,2,2,2,2)$ \\
\hline$C_{43,3}$ & $1,1,2,2,3,1,0,2,2,2,2,3,2,3,2,2,2,2,2,2,2)$ \\
\hline$C_{43,4}$ & $0,1,2,3,3,1,2,2,2,2,2,2,2,2,2,2,2,2,2,2,2)$ \\
\hline$C_{43,5}$ & $2,3,2,3,2,2,2,2)$ \\
\hline$C_{43,6}$ & $2,2,2,2,2,2,2,2)$ \\
\hline$C_{43,7}$ & $3,1,3,3,2,0,2,2)$ \\
\hline$C_{43,8}$ & $, 2,2,3,3,1,1,2,2)$ \\
\hline$C_{43,9}$ & $, 2,2,3,3,0,2,2,2)$ \\
\hline$C_{43,10}$ & $, 1,1,2,2,2,1,2,2$ \\
\hline$C_{48,1}$ & $2,3,3,3,2,3,3$ \\
\hline$C_{48,2}$ & $3,3,2,3, \quad$, \\
\hline$C_{48,3}$ & $0,2,2,2,2,2,3$ \\
\hline$C_{48,4}$ & $2,3,2,3,2,2,2,2)$ \\
\hline$C_{48,5}$ & $, 0,3,3,0,3)$ \\
\hline$C_{5}$ & $2,2,2$ \\
\hline$C_{5}$ & $, 3,3,3)$ \\
\hline$C_{52,3}$ & $3)$ \\
\hline$C_{52,4} \longrightarrow$ & $, 3,3,3,3,3)$ \\
\hline$C_{52,5}$ & $, 2,3,3,3)$ \\
\hline$C_{56}$ & $, 3,3$ \\
\hline$C_{56}$ & ) \\
\hline$C_{56,3}$ & $, 3,3,3,3,3)$ \\
\hline$C_{56,4}$ & $3,2,3,3,3,3)$ \\
\hline$C_{56,5}$ & $3,3,3,3,2,3,3,3)$ \\
\hline$C_{56,6}$ & $2,3,3,3,2,3,3,3)$ \\
\hline$C_{64,1}$ & $3,3,3,2,3,3,3)$ \\
\hline & $3,3,4,4)$ \\
\hline & $, 3,3,4,3,3)$ \\
\hline & $3,3,3,3,3,3,3,3)$ \\
\hline & $1,3,4,4,4,3,4,4)$ \\
\hline & $3,4,4,4,1,2,2,2)$ \\
\hline & $, 3,3,4,4,0,2,2,2)$ \\
\hline & $, 3,3,3,3,3,3,3,3)$ \\
\hline & $3,3,3,3,3,3,3,3)$ \\
\hline & $3,4,4,3,3,3,3,3)$ \\
\hline & 23323334 \\
\hline & $2,4,4,4,0,2,2,2)$ \\
\hline & $, 3,3,3,3,3)$ \\
\hline & $\left.\begin{array}{lll}3 & 3 & 3\end{array}\right)$ \\
\hline & \\
\hline
\end{tabular}


Table 5: Weight enumerators

\begin{tabular}{l|l|l|l}
\hline$W_{n, i}$ & \multicolumn{1}{|c|}{ Weight enumerator } & $W_{n, i}$ & \multicolumn{1}{c}{ Weight enumerator } \\
\hline$W_{36,1}$ & $1+48 y^{27}+12 y^{28}+3 y^{32}$ & $W_{36,2}$ & $1+45 y^{27}+15 y^{28}+3 y^{31}$ \\
\hline$W_{40,1}$ & $1+36 y^{30}+24 y^{31}+3 y^{32}$ & $W_{40,2}$ & $1+48 y^{30}+15 y^{32}$ \\
\hline$W_{43,1}$ & $1+45 y^{32}+15 y^{34}+3 y^{38}$ & $W_{43,2}$ & $1+39 y^{32}+24 y^{34}$ \\
$W_{43,3}$ & $1+39 y^{32}+12 y^{33}+12 y^{35}$ & $W_{43,4}$ & $1+27 y^{32}+24 y^{33}+12 y^{34}$ \\
$W_{43,5}$ & $1+42 y^{32}+18 y^{34}+3 y^{36}$ & $W_{43,6}$ & $1+15 y^{32}+48 y^{33}$ \\
$W_{43,7}$ & $1+54 y^{32}+6 y^{36}+3 y^{40}$ & $W_{43,8}$ & $1+51 y^{32}+12 y^{36}$ \\
$W_{43,9}$ & $1+51 y^{32}+12 y^{36}$ & $W_{43,10}$ & $1+63 y^{32}$ \\
\hline$W_{48,1}$ & $1+57 y^{36}+3 y^{40}+3 y^{44}$ & $W_{48,2}$ & $1+54 y^{36}+9 y^{40}$ \\
$W_{48,3}$ & $1+54 y^{36}+9 y^{40}$ & $W_{48,4}$ & $1+45 y^{36}+18 y^{38}$ \\
$W_{48,5}$ & $1+60 y^{36}+3 y^{48}$ & & \\
\hline$W_{52,1}$ & $1+42 y^{39}+15 y^{40}+6 y^{43}$ & $W_{52,2}$ & $1+45 y^{39}+12 y^{40}+3 y^{43}+3 y^{44}$ \\
$W_{52,3}$ & $1+48 y^{39}+12 y^{40}+3 y^{48}$ & $W_{52,4}$ & $1+45 y^{39}+15 y^{40}+3 y^{47}$ \\
$W_{52,5}$ & $1+48 y^{39}+9 y^{40}+6 y^{44}$ & & \\
\hline$W_{56,1}$ & $1+36 y^{42}+24 y^{43}+3 y^{48}$ & $W_{56,2}$ & $1+45 y^{42}+15 y^{44}+3 y^{46}$ \\
$W_{56,3}$ & $1+48 y^{42}+12 y^{44}+3 y^{48}$ & $W_{56,4}$ & $1+42 y^{42}+21 y^{44}$ \\
$W_{56,5}$ & $1+36 y^{42}+21 y^{43}+3 y^{44}+3 y^{47}$ & $W_{56,6}$ & $1+33 y^{42}+24 y^{43}+3 y^{44}+3 y^{46}$ \\
\hline$W_{64,1}$ & $1+51 y^{48}+12 y^{52}$ & $W_{64,2}$ & $1+51 y^{48}+12 y^{52}$ \\
$W_{64,3}$ & $1+51 y^{48}+12 y^{52}$ & $W_{64,4}$ & $1+15 y^{48}+48 y^{49}$ \\
$W_{64,5}$ & $1+57 y^{48}+3 y^{52}+3 y^{60}$ & $W_{64,6}$ & $1+54 y^{48}+6 y^{52}+3 y^{56}$ \\
$W_{64,7}$ & $1+54 y^{48}+6 y^{52}+3 y^{56}$ & $W_{64,8}$ & $1+27 y^{48}+24 y^{49}+12 y^{50}$ \\
$W_{64,9}$ & $1+39 y^{48}+12 y^{49}+12 y^{51}$ & $W_{64,10}$ & $1+45 y^{48}+15 y^{50}+3 y^{54}$ \\
$W_{64,11}$ & $1+42 y^{48}+18 y^{50}+3 y^{52}$ & $W_{64,12}$ & $1+57 y^{48}+6 y^{56}$ \\
$W_{64,13}$ & $1+63 y^{48}$ & $W_{64,14}$ & $1+39 y^{48}+24 y^{50}$ \\
$W_{64,15}$ & $1+60 y^{48}+3 y^{64}$ & & \\
\hline
\end{tabular}

An $[[n, k, d ; 0]]$ EAQECC is a standard quantum code. An $[[n, k, d ; n-k]]$ EAQECC is called maximal entanglement. If there is a quaternary Hermitian $\operatorname{LCD}[n, k, d]$ code, then there is a maximal entanglement $[[n, k, d ; n-k]]$ EAQECC (see e.g. [11] and [12]). From Proposition 5.4, there is a maximal entanglement $[[21 s+5,3,16 s+3 ; 21 s+2]]$ EAQECC for a positive integer $s$. It was shown in [9, Theorem 7] that $d \leq \frac{3 n \times 4^{k}}{4\left(4^{k}-1\right)}$ for an $[[n, k, d ; c]]$ EAQECC. Hence, a maximal entanglement [[21s $+5,3,16 s+3 ; 21 s+2]]$ EAQECC meets the above bound for a positive integer $s$. Therefore, the largest minimum weight among all maximal entanglement [[21s $+5,3, d ; 21 s+2]]$ EAQECC's is $16 s+3$ for a positive integer $s$. 
Table 6: Griesmer bound $\alpha_{4}(n, 3)$

\begin{tabular}{c|c|c|c|c|c}
\hline$n$ & $\alpha_{4}(n, 3)$ & $n$ & $\alpha_{4}(n, 3)$ & $n$ & $\alpha_{4}(n, 3)$ \\
\hline $21 s$ & $16 s$ & $21 s+7$ & $16 s+4$ & $21 s+14$ & $16 s+10$ \\
$21 s+1$ & $16 s$ & $21 s+8$ & $16 s+5$ & $21 s+15$ & $16 s+11$ \\
$21 s+2$ & $16 s$ & $21 s+9$ & $16 s+6$ & $21 s+16$ & $16 s+12$ \\
$21 s+3$ & $16 s+1$ & $21 s+10$ & $16 s+7$ & $21 s+17$ & $16 s+12$ \\
$21 s+4$ & $16 s+2$ & $21 s+11$ & $16 s+8$ & $21 s+18$ & $16 s+13$ \\
$21 s+5$ & $16 s+3$ & $21 s+12$ & $16 s+8$ & $21 s+19$ & $16 s+14$ \\
$21 s+6$ & $16 s+4$ & $21 s+13$ & $16 s+9$ & $21 s+20$ & $16 s+15$ \\
\hline
\end{tabular}

From [12, Tables 3 and 4] and Proposition 5.4, we have

$$
\begin{array}{ll}
d_{4}(21 s+2,3)=16 s, & d_{4}(21 s+3,3)=16 s+1 \\
d_{4}(21 s+4,3)=16 s+2, & d_{4}(21 s+5,3)=16 s+3,
\end{array}
$$

for a positive integer $s$, and

$$
\begin{array}{ll}
d_{4}(21 s+7,3)=16 s+4, & d_{4}(21 s+8,3)=16 s+5, \\
d_{4}(21 s+9,3)=16 s+6, & d_{4}(21 s+12,3)=16 s+8, \\
d_{4}(21 s+13,3)=16 s+9, & d_{4}(21 s+17,3)=16 s+12 \\
d_{4}(21 s+18,3)=16 s+13, &
\end{array}
$$

for a nonnegative integer $s$. In the remainder of this section, we consider the remaining cases.

As a special case of Theorem 3.4 (ii), we have the following:

Lemma 5.6. Suppose that

$$
\left(n_{0}, d_{0}\right) \in\left\{\begin{array}{l}
(16,12),(20,15),(32,24),(36,27),(40,30), \\
(48,36),(52,39),(56,42),(64,48)
\end{array}\right\} .
$$

If there is no quaternary Hermitian $L C D\left[n_{0}, 3, d_{0}\right]$ code $C$ with $d\left(C^{\perp_{H}}\right) \geq 2$. Then there is no quaternary Hermitian $L C D\left[21 s+n_{0}, 3,16 s+d_{0}\right]$ code $D$ with $d\left(D^{\perp_{H}}\right) \geq 2$ for a positive integer $s$.

Proposition 5.7. For a positive integer $s, d_{4}(21 s, 3)=16 s-1$.

Proof. By Theorem [3.3, $d_{4}(21 s, 3) \leq 16 s-1$. There is a quaternary Hermitian LCD $[21,3,15]$ code [12, Table 3]. By Lemma [5.3, there is a quaternary Hermitian LCD $[21 s, 3,16 s-1]$ code. 
The results in Table 1 are used in the following proposition.

Proposition 5.8. For a nonnegative integer s,

$$
\begin{aligned}
& d_{4}(21 s+11,3)=16 s+7, \quad d_{4}(21 s+16,3)=16 s+11 \\
& d_{4}(21 s+20,3)=16 s+14
\end{aligned}
$$

Proof. Suppose that $\left(n_{0}, d_{0}\right) \in\{(16,12),(20,15),(32,24)\}$ and $s$ is a nonnegative integer. From Table 1, there is no quaternary Hermitian LCD $\left[n_{0}, 3, d_{0}\right]$ code. Hence, by Lemma 5.6, there is no quaternary Hermitian LCD $\left[21 s+n_{0}, 3,16 s+d_{0}\right]$ code $C$ with $d\left(C^{\perp_{H}}\right) \geq 2$. Suppose that there is a quaternary Hermitian LCD $[n, 3, d]$ code $D$ with $d\left(D^{\perp_{H}}\right)=1$. By Lemma 2.2, a quaternary Hermitian LCD $[n-1,3, d]$ code is constructed. This contradicts the Griesmer bound (see Table 6).

It was shown in [12, Tables 3 and 4$]$ that

$$
\begin{aligned}
& d_{4}(21 s+11,3) \geq 16 s+7, \quad d_{4}(21 s+16,3) \geq 16 s+11 \\
& d_{4}(21 s+20,3) \geq 16 s+14 .
\end{aligned}
$$

From Table1, it is known that there is no quaternary Hermitian LCD $[11,3,8]$ code. This completes the proof.

The results in Proposition 4.1 are used in the following proposition.

Proposition 5.9. For a nonnegative integer s,

$$
\begin{array}{ll}
d_{4}(21 s+1,3)=16 s-1, & d_{4}(21 s+6,3)=16 s+3 \\
d_{4}(21 s+10,3)=16 s+6, & d_{4}(21 s+14,3)=16 s+9 \\
d_{4}(21 s+15,3)=16 s+10, & d_{4}(21 s+19,3)=16 s+13
\end{array}
$$

Proof. Suppose that $s$ is a nonnegative integer and

$$
\left(n_{0}, d_{0}\right) \in\{(36,27),(40,30),(48,36),(52,39),(56,42),(64,48)\} .
$$

By Proposition 4.1 (i), (iii)-(v), there is no quaternary Hermitian LCD $\left[n_{0}, 3, d_{0}\right]$ code. Hence, by Lemma 5.6, there is no quaternary Hermitian LCD $\left[21 s+n_{0}, 3,16 s+d_{0}\right]$ code $C$ with $d\left(C^{\perp_{H}}\right) \geq 2$. Now suppose that there is a quaternary Hermitian LCD $\left[21 s+n_{0}, 3,16 s+d_{0}\right]$ code $D$ with $d\left(D^{\perp_{H}}\right)=1$. By Lemma [2.2, a quaternary Hermitian LCD $\left[21 s+n_{0}-1,3,16 s+d_{0}-1\right]$ code is constructed. This contradicts $d_{4}(21 s, 3)=16 s-1$ in Proposition 5.7 
if $21 s+n_{0}=21 s+1(s \geq 1)$, and this contradicts the Griesmer bound (see Table 6) otherwise.

It was shown in [12, Tables 3 and 4] that

$$
\begin{array}{ll}
d_{4}(21 s+1,3) \geq 16 s-1, & d_{4}(21 s+6,3) \geq 16 s+3, \\
d_{4}(21 s+10,3) \geq 16 s+6, & d_{4}(21 s+14,3) \geq 16 s+9, \\
d_{4}(21 s+15,3) \geq 16 s+10, & d_{4}(21 s+19,3) \geq 16 s+13 .
\end{array}
$$

From Table 1 and Proposition 4.1 (ii), it is known that there is no quaternary Hermitian LCD $[n, 3, d]$ code for

$$
\begin{aligned}
(n, d)=(6,4),(10,7), & (14,10),(15,11), \\
& (19,14),(27,20),(22,16),(31,23),(35,26),(43,32) .
\end{aligned}
$$

This completes the proof.

Combining Propositions 5.4, 5.7, 5.8 and 5.9 with Remark 5.2, we determine $d_{4}(n, 3)$ as described in Table 7 and we complete the proof of Theorem 5.1 .

Table 7: $d_{4}(n, 3)(n \geq 6)$

\begin{tabular}{c|c|c|c|c|c}
\hline$n$ & $d_{4}(n, 3)$ & Reference & $n$ & $d_{4}(n, 3)$ & Reference \\
\hline $21 s$ & $16 s-1$ & Proposition 5.7] & $21 s+11$ & $16 s+7$ & Proposition [5.8 \\
$21 s+1$ & $16 s-1$ & Proposition 5.9] & $21 s+12$ & $16 s+8$ & [12, Table 4] \\
$21 s+2$ & $16 s$ & [12, Table 4] & $21 s+13$ & $16 s+9$ & [12, Table 4] \\
$21 s+3$ & $16 s+1$ & [12, Table 4] & $21 s+14$ & $16 s+9$ & Proposition 5.9] \\
$21 s+4$ & $16 s+2$ & [12, Table 4] & $21 s+15$ & $16 s+10$ & Proposition [5.9] \\
$21 s+5$ & $16 s+3$ & Proposition [5.4 & $21 s+16$ & $16 s+11$ & Proposition [5.8 \\
$21 s+6$ & $16 s+3$ & Proposition [5.9] & $21 s+17$ & $16 s+12$ & [12, Table 4] \\
$21 s+7$ & $16 s+4$ & [12, Table 4] & $21 s+18$ & $16 s+13$ & [12, Table 4] \\
$21 s+8$ & $16 s+5$ & [12, Table 4] & $21 s+19$ & $16 s+13$ & Proposition [5.9] \\
$21 s+9$ & $16 s+6$ & [12, Table 4] & $21 s+20$ & $16 s+14$ & Proposition [5.8 \\
$21 s+10$ & $16 s+6$ & Proposition [5.9] & & & \\
\hline
\end{tabular}

Acknowledgment. This work was supported by JSPS KAKENHI Grant Number 15H03633. The authors would like to thank the anonymous referees for the useful comments. 


\section{References}

[1] M. Araya and M. Harada, On the classification of linear complementary dual codes, Discrete Math. 342 (2019), 270-278.

[2] W. Bosma, J. Cannon and C. Playoust, The Magma algebra system I: The user language, J. Symbolic Comput. 24 (1997), 235-265.

[3] I. Bouyukliev, M. Grassl and Z. Varbanov, New bounds for $n_{4}(k, d)$ and classification of some optimal codes over GF(4), Discrete Math. 281 (2004), 43-66.

[4] P.J. Cameron and J.H. van Lint, Designs, Graphs, Codes and Their Links, Cambridge University Press, Cambridge, 1991.

[5] C. Carlet, S. Mesnager, C. Tang and Y. Qi, New characterization and parametrization of LCD codes, IEEE Trans. Inform. Theory 65 (2019), 39-49.

[6] C. Carlet, S. Mesnager, C. Tang, Y. Qi and R. Pellikaan, Linear codes over $\mathbb{F}_{q}$ are equivalent to LCD codes for $q>3$, IEEE Trans. Inform. Theory 64 (2018), 3010-3017.

[7] M. Grassl, Code tables: Bounds on the parameters of various types of codes, Available online at http://www.codetables.de/, Accessed on 2019-02-12.

[8] C. Güneri, B. Özkaya and P. Solé, Quasi-cyclic complementary dual codes, Finite Fields Appl. 42 (2016), 67-80.

[9] C.-Y. Lai, T.A. Brun and M.M. Wilde, Dualities and identities for entanglement-assisted quantum codes, Quantum Inf. Process. 13 (2014), 957-990.

[10] R. Li, Research on quantum codes and self-orthogonal codes, Postdoctor work report, Xi'an Jiaotong University (2008).

[11] R. Li, X. Li and L. Guo, On entanglement-assisted quantum codes achieving the entanglement-assisted Griesmer bound, Quantum Inf. Process. 14 (2015), 4427-4447. 
[12] L. Lu, R. Li, L. Guo and Q. Fu, Maximal entanglement entanglementassisted quantum codes constructed from linear codes, Quantum Inf. Process. 14 (2015), 165-182.

[13] F.J. MacWilliams, A.M. Odlyzko, N.J.A. Sloane and H.N. Ward, Selfdual codes over GF(4), J. Combin. Theory Ser. A 25 (1978), 288-318.

[14] J.L. Massey, Linear codes with complementary duals, Discrete Math. 106/107 (1992), 337-342.

\section{Appendix}

In Appendix, we give a proof of Proposition 2.4. For $a=\left(a_{1}, a_{2}, \ldots, a_{5}\right) \in$ $\mathbb{Z}_{\geq 0}^{5}$, we define a quaternary $[n, 2]$ code $C(a)$ having generator matrix of the form $G(a)=\left(\begin{array}{ll}I_{2} & M(a)\end{array}\right)$, where

$$
M(a)=\left(\begin{array}{ccccc}
\mathbf{0}_{a_{1}} & \mathbf{1}_{a_{2}} & \mathbf{1}_{a_{3}} & \mathbf{1}_{a_{4}} & \mathbf{1}_{a_{5}} \\
\mathbf{1}_{a_{1}} & \mathbf{0}_{a_{2}} & \mathbf{1}_{a_{3}} & \omega \mathbf{1}_{a_{4}} & \omega^{2} \mathbf{1}_{a_{5}}
\end{array}\right) .
$$

It is trivial that any quaternary $[n, 2]$ code $C$ is equivalent to some $C(a)$ if $d\left(C^{\perp_{H}}\right) \geq 2$. By considering all codewords, the weight enumerator of the code $C(a)$ is written using $a_{1}, a_{2}, \ldots, a_{5}$ as follows:

$$
\begin{aligned}
& 1+3 y^{1+a_{1}+a_{3}+a_{4}+a_{5}}+3 y^{1+a_{2}+a_{3}+a_{4}+a_{5}} \\
& +3 y^{2+a_{1}+a_{2}+a_{4}+a_{5}}+3 y^{2+a_{1}+a_{2}+a_{3}+a_{4}}+3 y^{2+a_{1}+a_{2}+a_{3}+a_{5}} .
\end{aligned}
$$

The matrix $G(a) \overline{G(a)}^{T}$ is written using $a_{1}, a_{2}, \ldots, a_{5}$ as follows:

$$
\left(\begin{array}{cc}
1+a_{2}+a_{3}+a_{4}+a_{5} & a_{3}+\omega a_{4}+\omega^{2} a_{5} \\
a_{3}+\omega^{2} a_{4}+\omega a_{5} & 1+a_{1}+a_{3}+a_{4}+a_{5}
\end{array}\right)
$$

Hence, the determinant of $G(a) \overline{G(a)}^{T}$ is written using $a_{1}, a_{2}, \ldots, a_{5}$ as follows:

$$
\begin{aligned}
& 1+a_{1}+a_{2}+a_{1} a_{2}+a_{1} a_{3}+a_{1} a_{4}+a_{1} a_{5}+a_{2} a_{3}+a_{2} a_{4}+a_{2} a_{5} \\
& +\left(\omega+\omega^{2}\right)\left(a_{3} a_{4}+a_{3} a_{5}+a_{4} a_{5}\right) .
\end{aligned}
$$

Lemma 5.10. Suppose that $n \equiv 0,4(\bmod 5)$. If there is a quaternary Hermitian $L C D\left[n, 2,\left\lfloor\frac{4 n}{5}\right\rfloor\right]$ code $C$, then $d\left(C^{\perp_{H}}\right) \geq 2$. 
Proof. Suppose that $n \equiv 0,4(\bmod 5)$. Suppose that there is a quaternary Hermitian LCD $\left[n, 2,\left\lfloor\frac{4 n}{5}\right\rfloor\right]$ code $C$ with $d\left(C^{\perp_{H}}\right)=1$. By Lemma 2.2, a quaternary Hermitian LCD $\left[n-1,2,\left\lfloor\frac{4 n}{5}\right\rfloor\right]$ code is constructed. This contradicts (3)).

Suppose that $n \equiv 0,4(\bmod 5)$. Let $C$ be a quaternary Hermitian LCD $\left[n, 2,\left\lfloor\frac{4 n}{5}\right\rfloor\right]$ code. By Lemma [5.10, we may assume without loss of generality that $C=C(a)$, that is, $C$ has generator matrix of the following form $G(a)=$ $\left(\begin{array}{ll}I_{2} & M(a)\end{array}\right)$, where $M(a)$ is listed in (77). From the length and the minimum weight of $C(a), a_{1}, a_{2}, \ldots, a_{5}$ satisfy the following conditions:

$$
\begin{aligned}
1+\sum_{i \in\{1,2,3,4,5\} \backslash\{j\}} a_{i} & \geq\left\lfloor\frac{4 n}{5}\right\rfloor \quad(j=1,2), \\
2+\sum_{i \in\{1,2,3,4,5\} \backslash\{j\}} a_{i} & \geq\left\lfloor\frac{4 n}{5}\right\rfloor(j=3,4,5), \\
2+\sum_{i \in\{1,2,3,4,5\}} a_{i} & =n .
\end{aligned}
$$

From (9)-(11), we have

$$
\begin{aligned}
a_{i}+1 & \leq n-\left\lfloor\frac{4 n}{5}\right\rfloor \quad(i=1,2), \\
a_{i} & \leq n-\left\lfloor\frac{4 n}{5}\right\rfloor \quad(i=3,4,5) .
\end{aligned}
$$

- Suppose that $n=5 \mathrm{~s}$. From (12), we have

$$
a_{i} \leq s-1(i=1,2) \text { and } a_{i} \leq s(i=3,4,5) .
$$

Then we have

$$
n=2+a_{1}+a_{2}+a_{3}+a_{4}+a_{5} \leq 5 s=n .
$$

Hence, we have

$$
a_{1}=a_{2}=s-1 \text { and } a_{3}=a_{4}=a_{5}=s .
$$

By (8)), using $s$, the determinant of $G(a) \overline{G(a)}^{T}$ is written as $10 s^{2}-6 s$. Hence, $C(a)$ is not Hermitian LCD for every positive integer $s$. 
- Suppose that $n=5 s+4$. From (12), we have

$$
a_{i} \leq s(i=1,2) \text { and } a_{i} \leq s+1(i=3,4,5) .
$$

Then we have

$$
n=2+a_{1}+a_{2}+a_{3}+a_{4}+a_{5} \leq 5 s+5=n+1 .
$$

Hence, we have

$$
\begin{array}{r}
\left|\left\{i \in\{1,2\} \mid a_{i} \leq s-2\right\}\right|=\left|\left\{i \in\{3,4,5\} \mid a_{i} \leq s-1\right\}\right|=0 \\
\left|\left\{i \in\{1,2\} \mid a_{i}=s-1\right\}\right|+\left|\left\{i \in\{3,4,5\} \mid a_{i}=s\right\}\right|=1 .
\end{array}
$$

This yields that there are the five possibilities for $a=\left(a_{1}, a_{2}, a_{3}, a_{4}, a_{5}\right)$, where the results are listed in Table 8 . The determinant det of $G(a) \overline{G(a)}^{T}$ is also listed in Table 8 . Therefore, $C_{i}(i=1,2, \ldots, 5)$ is not Hermitian LCD for every positive integer $s$.

This completes the proof of Proposition 2.4.

Table 8: Case $n=5 s+4$

\begin{tabular}{c|c|c}
\hline$C(a)$ & $a=\left(a_{1}, a_{2}, a_{3}, a_{4}, a_{5}\right)$ & \multicolumn{1}{c}{$\operatorname{det}$} \\
\hline$C_{1}$ & $(s-1, s, s+1, s+1, s+1)$ & $10 s^{2}+10 s$ \\
$C_{2}$ & $(s, s-1, s+1, s+1, s+1)$ & $10 s^{2}+10 s$ \\
$C_{3}$ & $(s, s, s, s+1, s+1)$ & $10 s^{2}+10 s+2$ \\
$C_{4}$ & $(s, s, s+1, s, s+1)$ & $10 s^{2}+10 s+2$ \\
$C_{5}$ & $(s, s, s+1, s+1, s)$ & $10 s^{2}+10 s+2$ \\
\hline
\end{tabular}

